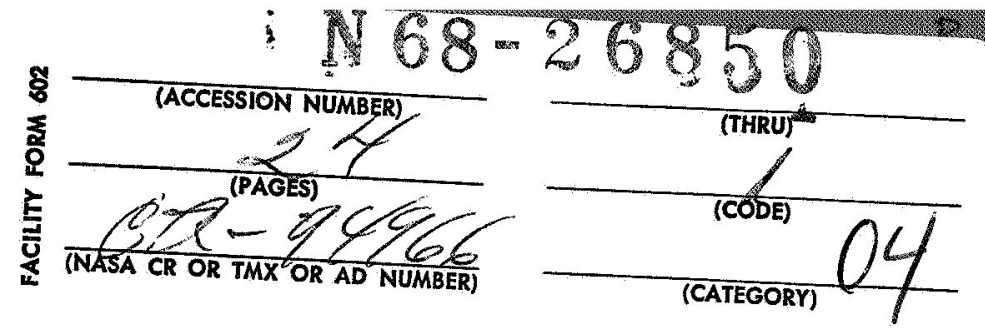

-

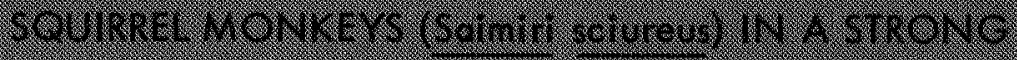

s. 1 . E.

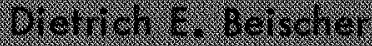

MVIA JOINT REPORT

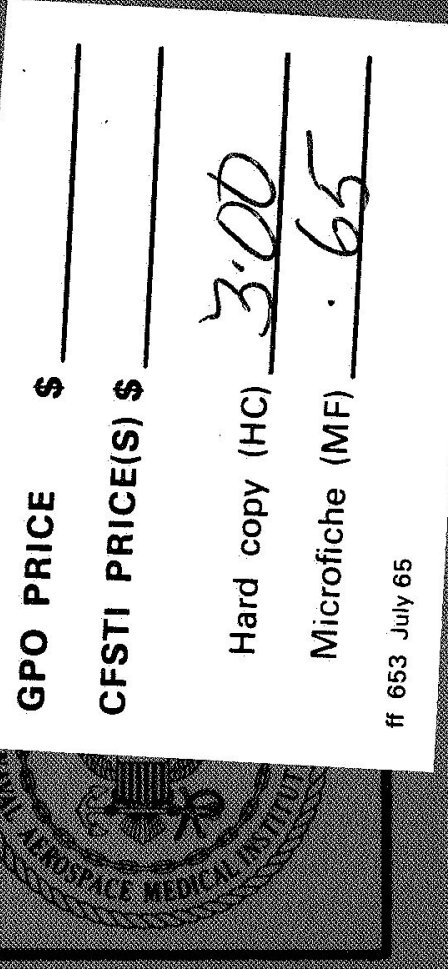

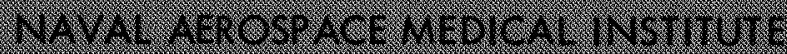

r.

(4)

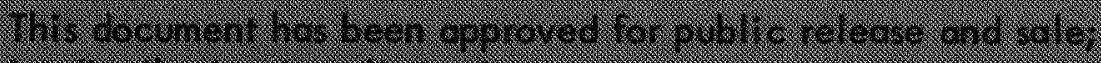

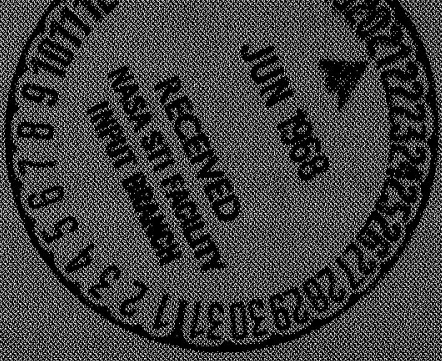

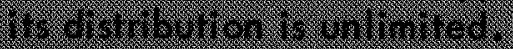


This document has been approved for public release and sale; its distribution is unlimited.

\title{
VECTORCARDIOGRAM AND AORTIC BLOOD FLOW OF SQUIRREL MONKEYS (Saimiri sciureus) IN A STRONG SUPERCONDUCTIVE ELECTROMAGNET*
}

Dietrich E. Beischer

\author{
Bureau of Medicine and Surgery \\ MR005.04-0029.12 \\ NASA Order R-39
}

Approved by

Ashton Graybiel, M.D. Head, Research Department
Released by

Captain J. W. Weaver, MC USN Commanding Officer

\section{April 1968}

* This research was conducted under the sponsorship of the Office of Biotechnology and Human Research, National Aeronautics and Space Administration. 
SUMMARY PAGE

\section{THE PROBLEM}

Magnetohydrodynamic power generation and other uses of strong magnetic fields, for instance in connection with lasers, are expected to expose man in the near future for long periods of time to strong magnetic fields. Since little is known about the physiological effects of such fields on man, exploratory studies with subhuman primates appear to be most desirable.

\section{FINDINGS}

Vectorcardiograms of two squirrel monkeys in a superconductive magnet with a field strength up to 100,000 Oe were evaluated. A pulsating electrical dipole (flowgram) was generated by movement of blood in the magnetic field and was superimposed on the ECG. This flowgram allowed observation of cardiac blood flow and determination of the velocity of blood in the aorta. The flow electromotive force does not interfere with cardiac activity during short-time exposure, but during long-time exposure the heart rate is reduced.

\section{ACKNOWLEDGMENTS}

Grateful acknowledgment is made to the staff of the Magnetics and Cryophysics Branch, Lewis Research Center, National Aeronautics and Space Administration, Cleveland, Ohio, for the use and the operation of the superconductive magnet. In particular, the cooperation of Mr. James $\mathrm{C}$. Laurence and $\mathrm{Mr} . \mathrm{W}$. D. Coles is highly appreciated. A number of colleagues of the Naval Aerospace Medical Institute contributed advice and help which are greatly appreciated: Mr. C. S. Ezell and Mr. E. G. King assisted in the experiments and helped in the evaluation; Dr. H. H. Khalil and Dr. S. P. Pakes contributed special studies; Mrs. M. P. Boss took excellent care of the animals.

Experiments reported herein were conducted according to the principles enunciated in "Guide for Laboratory Animal Facilities and Care" prepared by the Committee on the Guide for Laboratory Animal Resources, National Academy of Sciences - National Research Council. 


\section{INTRODUCTION}

In a previous study by Beischer and Knepton (1), wherein squirrel monkeys were exposed to magnetic fields up to $70,000 \mathrm{Oe}$, a striking increase in the $\mathrm{T}$ wave amplitude of the electrocardiogram (ECG) was observed and found to be proportional to the field strength, with a value of $0.05 \mathrm{mV}$ per 10,000 Oe. An influence of the magnetic field on the repolarization of the heart was advanced as a tentative interpretation of this observation. Recently, Togawa et al. (2) showed, in experiments with rabbits in a field of $10,000 \mathrm{Oe}$, that the electromotive force (emf) of blood flow was superimposed on the ECG. The emf of flow was small, but at least two peaks were observed between the $S$ wave and the end of the $T$ wave of the ECG. The connection of this potential with blood flow was demonstrated by reversal of the sign of the flow emf at inversion of the polarity of the magnetic field. In the present study with squirrel monkeys, the use of a superconductive magnet with high-field strength and application of vectorcardiographic leads furnished much stronger and clearer signals than those of the previous studies $(1,2)$. The observed increase of the $T$ wave has thus been confirmed to be a superimposition on the ECG of the emf generated by blood flow, as first suggested by Togawa. The magnetic method provides, in a single record, information on the electrical and mechanical activity of the heart and represents a new, noninvasive method of studying cardiac performance.

\section{APPARATUS AND PROCEDURE}

A superconductive magnet built by Radio Corporation of America was made available by the Magnetics and Cryophysics Branch, National Aeronautics and Space Administration, Lewis Research Center, Cleveland, Ohio (3), and was operated by personnel of that Center. The large snow-covered cylindrical container, the nitrogen Dewar, enclosed the helium Dewar with the magnetic coil (Figure $1^{*}$ ). The magnet itself was about $13.5 \mathrm{in}$. high, with an outside diameter of about $20 \mathrm{in}$. and an inside bore of $6 \mathrm{in}$. It consisted of 22 modules, or subcoils, and energy sinks, and allowed generation of a maximum field strength of 150,000 Oe in the center of the coil; up to 100,000 Oe fields only were used in the present experiment. To generate a field of this dimension and strength in a water-cooled magnet requires several megawatts of power and extensive cooling facilities. The field-distribution curve along the axis of the superconductive magnet had a bell-shape form, with the field strength constant to less than 1 per cent in a space of $1 \mathrm{cu}$ in., cocentered with the center of the magnetic field. A finger Dewar of $4.5 \mathrm{in}$. inner core diameter provided access at room temperature to the magnetic field. The maximum of the field was located $90.5 \mathrm{in}$. below the top of the finger Dewar. An automatically regulated heating coil in the lower part of the finger Dewar held the temperature in the experimental space constant at $25^{\circ} \mathrm{C}$.

*In order not to break the continuity of the text, all illustrations appear at the end of the report (pp 14 to 21 ). 
Two healthy, adult squirrel monkeys, "gothic arch" race Saimiri from Leticia, Brazil, held at the Institute colony for more than a year prior to the experiments, were

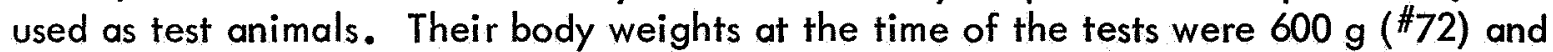
$708 \mathrm{~g}(\mathrm{MJ})$, respectively. Electrode placement followed the system described by Frank (4) for human clinical vectorcardiography (Figure 2). The electrodes were small silver cups of $6 \mathrm{~mm}$ diameter (Grass EEG electrodes). A drop of solder was applied to the concave side and shaped to form a knob which fitted in the holes of a rubber band. A small plastic sponge, freshly soaked with Sanborn Redux electrode paste, was placed in the cup of the electrode. The animals were prepared by shearing a band of pelt around the chest, the lower extremities between knee and ankle, and the dorsal part of the head. The five electrodes with Frank designations I, E, C, A, and M were placed in proper position by insertion of their knobs into the holes of the rubber band fastened around the thorax of the animal below the nipples. The remaining three electrodes were placed on the left leg (F) (between knee and ankle), right leg (ground), and on the head $(\mathrm{H})$, and fixed by adhesive tape. Great care was taken in placement of the electrodes since, due to the small size of the animal, small variations might have introduced errors in the measurements. During placement of the electrodes the nonanesthetized animal was held by an assistant, then placed into a contour-fitted Fiberglas container $11 \mathrm{in}$. long and $37 / 8 \mathrm{in}$. in diameter, and secured by Velcro bands. The container with the animal was attached to a long wooden rod which was used to insert the animal into the magnet and as a solid support for the leads. The leads were shielded by grounded nonferromagnetic metal shields extending nearly all the way from the electrodes to the lead selector, leaving only 4-in. lead sections close to the electrodes unshielded. To reduce artifacts the leads were tightly fastened to the container and to the insertion rod. An effort was made to avoid loops in the leads in order to reduce artifacts due to movement of the leads in the strong magnetic field.

The leads were connected to a Frank Lead Selector (Model 461-162B Sanborn, Waltham, Mass.). A Sanborn High Gain Amplifier (350-2700) and a Visicorder Oscillograph (Model 1108 Honeywell, Denver, Colorado) were used to amplify and record the biosignal. After calibration of the three channels with a $1 \mathrm{mV}$ test signal, a control vectorcardiogram was recorded with the animal outside the magnetic field. The monkey was then inserted into the field by lowering it at a velocity of about 1 in. per second into the magnet operated at the desired field strength. The heart of the monkey was located in the $1 \mathrm{cu}$ in. space of homogeneous field strength. After the vectorcardiogram was recorded the animal was retracted from the field and another measurement made. Changes of the field strength of the superconductive magnet could be made only slowly, and measurements at the three field strengths used in the experiment $(40,65$, and $100 \mathrm{kOe})$ were performed on three successive days, using the two squirrel monkeys as experimental subjects each day in at least five insertions each. Samples of tracings recorded with this procedure are shown in Figure $3 A$ (control) and 3B (magnetic field of $100,000 \mathrm{Oe}$ ). Details of the third complex of the $X$ tracing in Figure $3 \mathrm{~B}$ are seen in Figure 4.

The heart rate was determined from the ECG by counting the number of QRS complexes falling within $3 \mathrm{sec}$, and respiratory rate was derived from the 
frequency of shift of the base line. Figure $3 \mathrm{~A}$ and $\mathrm{B}$ show about one respiratory cycle each.

From the three values of the $X, Y$, and $Z$ traces of the vectorcardiogram (Figure 3) triaxial displays of ECG's were constructed for the time period following the QRS complex. Figure 5 shows as an example the spatial configuration of the $T$ wave of the control vectorcardiogram in comparison with the corresponding section constructed from the values of the exposed animal (Total of Figure 5). By vector subtraction of the control $T$ values from the Total values, the effect of the magnetic field could be demonstrated; this vectorgram was designated Flow in Figure 5. Peak magnitudes and directions of the $T$ as well as Flow vectors were derived from such three-dimensional displays.

The dimensions and the anatomic position of the aorta were determined by two different procedures. Open-chest caliper measurements of the aorta were made in the deeply anethesized animal. These measurements were supplemented by aortograms photographed after injection into the heart of 0.1 Hypaque - M 75\% Brand (Wintrop Laboratories, New York, New York). An aortogram of a squirrel monkey (nonexposed subject weighing $710 \mathrm{~g}$ ) is presented in Figure 6 . The caliper measurements were performed in two and the aortograms in four nonexposed monkeys. These six monkeys were comparable in weight and size with the animals used in the magnetic exposure experiments.

\section{RESULTS}

Even a casual glance at the tracings presented in Figure 3 revealed major effects of the strong magnetic field on the ECG, visible clearly in the complex following the QRS wave. The T wave was completely overshadowed by the strong signal generated by the aortic blood flow in the magnetic field. Subtraction of the control values from the field values, using a single tracing like the $X$ tracing in Figure 3 or the threedimensional display (Figure 5), allowed characterization of the flow potential in great detail.

The characteristics of the flow potential were especially well manifested in the $X$ tracing in Figure $3 B$, as compared to the control tracing (Figure $3 A$ ) where the complex following QRS deviated little from the baseline. The flow signal had a fast ascending and a gradually descending branch, characteristic of graphs for the aortic blood velocity as measured by a flowmeter on the aorta. The main spatial direction of the flow potential can be derived from displays like that of Figure 5. The vector of the flow potential during its total duration was roughly pointing in the direction of the $+X$ axis with a slight anterior inclination. This direction was predominant in both exposed animals and was observed in all experiments at the different field strengths.

The maximum scalar value of the flow potential was linearly related to the strength of the magnetic field. Maxima of flow potentials measured in fields of 40,000, 65,000 and 100,000 Oe are plotted in Figure 7. These potentials were considerably influenced by the respiratory cycle; the values plotted in Figure 7 
represent the mean of the highest value measured at each field strength in 25 respiratory cycles during inspiration and the mean of the lowest values in the same number of cycles measured during expiration. The respiratory periods are well defined by the baseline shifts as seen best in the $Z$ trace of Figure 3B. A respiratory rate of about 50 to 60 cycles was observed in the control measurements; this rate remained the same during exposure of the animals to the different magnetic field strengths.

Superimposed on the ascending and descending branch of the flow potential, some interesting details were noticed. At certain time intervals the $X, Y$, and $Z$ tracings showed well-defined peaks and notches (Figure 3B) which carry letters from $A$ through $H$ in the single complex seen in Figure 4. Their recurrence at regular time intervals in cycle after cycle was so typical that these singular points of the potential curve must represent characteristic events in the course of the blood flow. Since these events were expressed at the same time in all leads $(X, Y$, and $Z$ ), they could not have been artifacts in a single lead. The notches in the flow curve were seen in the records of both monkeys at all field strengths studied; however, they were expressed more in the records obtained at 100,000 Oe. Table I presents a characteristic example of the time intervals between singular points of Figure 4.

Table I

Time Intervals Between Lettered Events in Figure 4

\begin{tabular}{ll}
\hline Events & $\frac{\text { Second }}{0.010}$ \\
\hline A to $B$ & 0.005 \\
$B$ to $C$ & 0.015 \\
C to $D$ & 0.030 \\
$D$ to $E$ & 0.110 \\
$B$ to $F$ & 0.007 \\
$F$ to $G$ & 0.240 (heart rate 250) \\
$Q$ to $Q$ & 0.028 \\
$Q$ to $(S)$ & \\
\hline
\end{tabular}


These time intervals were determined with an accuracy of about 1 millisecond. The accuracy of measurement was especially high at points where the curve changed abruptly (points A, B, and C) and depended on the width of the recording trace.

The configuration of the electrocardiograms of squirrel monkeys in strong magnetic fields was so much distorted by the flow potential that values for some complexes and intervals were difficult to determine. The following results will provide material for discussing the question of whether the flow potential is the only factor modifying the ECG tracings or if a direct influence of the strong field on the electrical processes in the heart can be demonstrated.

In the present study the exposure period to the high magnetic field was so short, less than 15 minutes, that the slowly developing decrease in the heart rate and increase in the degree of sinus arrhythmia which were previously described (1) were not noticed. Heart rates of the two squirrel monkeys ranged between 240 and 280 . Because the rate was not always the same in the control and experimental measurements, the rate should be considered in comparison of complexes and intervals of the ECG's.

The $P$ wave in the control record (Figure $3 A$ ) had a small amplitude of about 0.1 millivolt in the $X$ trace and was scarcely noticeable in the other two traces. Thus, the $P$ vector extended mainly in the $+X$ axis, with a slight inclination towards the $+Y$ axis. The duration of the normal $P$ wave in the squirrel monkey was about $0.02 \mathrm{sec}$, and the $P-R$ interval in the control measurement was about $0.045 \mathrm{sec}$.

The $\mathrm{P}$ wave of the monkeys in the strong magnetic field (Figure 3B) appeared to be influenced by the field, as the amplitude and duration showed variations with respiration. In expiration the amplitude of $P$ was similar to that of control conditions $(0.1 \mathrm{mV})$ and increased during inspiration to about $0.3 \mathrm{mV}$ in the $X$ tracing. The direction of the vector of the $P$ wave was not changed from that of control conditions. The duration of the wave varied between $0.02 \mathrm{sec}$ and $0.05 \mathrm{sec}$, and the $P-R$ interval between $0.045 \mathrm{sec}$ in expiration and $0.06 \mathrm{sec}$ in inspiration. The $P$ wave in the magnetic field was superimposed on a stronger flow signal that started to increase earlier than the control $P$ wave and lasted longer. This flow signal was most likely connected with venous return and the filling of the atrium. It was directed in the $+Z$ axis of the animal (posteriorly).

The QRS complex in the control tracing (Figure $3 \mathrm{~A}$ ) had an average duration of $0.035 \mathrm{sec}$. The electric axis of the QRS complex was directed to the left $\left(+30^{\circ}\right)$, anteriorly, indicating a vertical heart position. The strongest signal was observed in the $X$ tracing $(+1.6 \mathrm{mV})$; next in intensity was the signal in the $Z$ tracing with -0.8 $\mathrm{mV}$, and then the signcl in the $Y$ tracing $(+0.35 \mathrm{mV})$. In the strongest field $(100,000$ $\mathrm{Oe}$ ) (Figure 3B) neither duration, axis, nor amplitude of the QRS complex was changed. This observation holds true for both animals at all. field strengths tested and is in agreement with earlier results (1). 
The axis of the $T$ wave in the control measurement (Figure 3A) was directed anteriorly $\left(+90^{\circ}\right)$ and slightly inferiorly $\left(10^{\circ}\right)$. Amplitude of the $T$ in the $Z$ tracing of Figure $3 \mathrm{~A}$ was $0.3 \mathrm{mV}$. The $\mathrm{S}-\mathrm{T}$ segment was about $0.03 \mathrm{sec}$, and the $Q-T$ interval was $0.15 \mathrm{sec}$.

The $T$ wave in the strongest magnetic field (Figure $3 B$ ) is seen as a peak superimposed on the flow potential curve and is marked in Figure 4 as such. The three traces in Figure 3B demonstrate that the $T$ wave was directed anteriorly as in the control tracing and had probably the same amplitude, but accurate measurements were not possible due to the predominant flow potential. The S-T segment could not be determined, but the time interval from $Q$ to $T$ (peak) could be measured with good accuracy and was the same as in the control record.

Notch $F$ and peak $G$ in Figure 4 were most probably connected with the closing of the aortic valve. The $Q-F$ interval was about $0.16 \mathrm{sec}$ (average of 10 measurements), and the time interval from point $B$ to point $F$ (points representing opening and closing of the aortic valve, respectively), $0.11 \mathrm{sec}$.

Aortogram measurements in three monkeys showed a mean diameter of the ascending branch of the aorta close to the root of $5 \mathrm{~mm}$. The mean length of this branch from the root to the culmination of the aortic arch in the same animals was $20 \mathrm{~mm}$. These measurements hold true for a body weight of about $700 \mathrm{~g}$ in the squirrel monkey.

\section{DISCUSSION}

\section{SUPERCONDUCTIVE MAGNET IN ELECTROPHYSIOLOGY}

A superconductive magnet was used for the first time in VCG studies of primates in very high magnetic fields. Squirrel monkeys had previously been exposed to very high magnetic fields in modified Bitter magnets $(1,5)$, but experimental difficulties were experienced in ECG and EEG measurements. Ripple current from the generator and mechanical vibration were recognized as artifact producing factors. A certain amount of ripple current could not be avoided in a magnet of low inductance supplied by a mechanical generator and it interfered with electrophysiological measurements. The strong stream of cooling water in the Bitter magnet caused vibrations which moved the magnetic field relative to a stationary subject and probably exposed it to an alternating magnetic field. The enormous power consumption in the megawatt range characteristic of the Bitter magnet limited the exposure period to economically feasible time periods and prohibited long-term physiological observations. These adverse factors were absent in experiments with the superconductive magnet. Such magnets can be operated free of ripple current and vibration and need only comparatively small power generators. During operation in the persistant mode the superconductive magnet does not need an outside power supply, although the cost of liquid helium which can be reduced by a regeneration facility may be a limiting factor for extended operation. 
All things considered, the use of superconductive magnets in generation of high magnetic fields for electrophysiological experimentation is highly recommended.

Difficulties which were inherent to electrophysiological measurements in strong magnetic fields remain to be solved. The electrical leads in connection with the animal body formed a conductor loop, and a change of the magnetic flux density in this loop induced an electromotive force in the conductor. This force is proportional to the flux and to the mode of change of the loop area; in a field of 100,000 Oe an area change of $1 \mathrm{~cm}^{2}$ will induce about $1 \mathrm{mV}$ in the conductor loop. It was expected that respiratory, cardiac, and other movements would open and close the conductor loop to a certain extent. To counteract the effect of such movements the lead wires were placed as parallel as possible to the magnetic flux and solidly attached to the animal container and to the insertion rod. An estimate of the electromotive force induced by change of the chest diameter during respiration could be made by comparing the baseline amplitudes in the field with the corresponding control data. The maximum amplitude in the field was less than $0.1 \mathrm{mV}$ larger than the maximum control amplitude. Cardiac action through chest diameter change was expected to induce a still smaller voltage in the lead loop. Thus, under the given experimental conditions the electromotive force produced by motion of the lead conductor in the magnetic field may be disregarded at least as far as the ECG is concerned.

\section{FRANK LEAD SYSTEM FOR SMALL PRIMATES}

Application of the Frank (4) lead system for spatial vectorcardiography on squirrel monkeys needs some comment. This lead system with orthogonal image vectors projected onto anatomical body axes was developed specifically for the torso shape of man and has found extensive clinical use. Scaling this system down to a small primate like the squirrel monkey would require careful analysis, and the experiments of Frank should have been repeated with an accurate three-dimensional homogeneous torso model of the squirrel monkey. However, such an extensive investigation was not feasible within the limits of the present study; therefore, it was decided to apply the Frank lead system in its present form to the squirrel monkey and judge its merits from the results. The record sample in Figure 3A of a normal squirrel monkey indicated that the QRS complex as well as the configuration of the $P$ and $T$ wave was similar to records received from normal man in a sitting position, and no gross distortions of amplitude or vector direction were noted.

Theoretically, no flow potential should have been recorded in the $Y$ tracing since the body axis was parallel to the direction of the magnetic field. However, the recording of the $Y$ trace (Figure 3B) shows a trace which at first glance appears to contain electronic interference but which actually registers events also seen in the other two traces. This is a clear indication that the resistor proportions selected by Frank for man should have been corrected for use in the squirrel monkey. Later discussion will show that the vector of the flow potential derived from the $X$ and $Z$ tracings had, in general, the expected spatial orientation in relation to the known orientation of the aorta, which justifies the application of the Frank lead system in monkeys in first approximation. 
Incidentally, the use of a strong magnetic field would also allow a sensitive test of the validity of the selection of resistors in the Frank lead system in its use for measurement of the vectorcardiogram in man. If the resistors have been selected correctly no flow signals should be observed in a tracing with its axis parallel to the field direction.

\section{INTERPRETATION OF FLOW POTENTIAL}

\section{General Principles}

The electromotive force registered immediately following the QRS complex was generated by blood flowing in the presence of a magnetic field. The vector of this electric field is directed-perpendicularly to the direction of the magnetic field as well as the direction of the movement of the blood. The polar direction of the emf is found by applying the right-hand rule. In the present study the magnetic field was applied parallel to the long axis of the animal; thus, all blood flow in the direction of the animal long axis did not generate an emf, and only the component of blood flow directed perpendicularly to the magnetic field contributed to the emf. In the case of the aorta, an emf was generated by blood flowing through the curving aorta to the degree to which the ascending branch deviated from the direction of the body axis. The maximum was reached when the blood passed the peak of the arch where the flow is perpendicular to the magnetic field. As the descending branch approached a direction parallel to the field and the body axis, the electrical signal diminished and reached zero when complete parallelism was achieved.

In a discussion of the flow signal it should be kept in mind that the technique of using external electrodes rendered at any given time the vector sum of the flow potentials generated throughout the body. Under certain conditions branching flow, as in the pulmonary arteries, will generate in the branches electromotive forces which are equal in intensity but opposite in sign, and the two signals will cancel at some distance from the sources. No signal from such branching flow was expected to reach the external electrodes. This distinguished the present experimental method from the magnetic flowmeter in which a cuff is applied with electrodes positioned locally on the vessel wall and in which the flow in this specific vessel is determined by measurement of the emf generated in a magnetic field.

The present method, by successive application of the magnetic field parallel to the three orthogonal axes of the animal's body, would provide a complete picture of the direction and velocity of blood flow in the animal's body. Reversal of the field direction, as used in Togawa's experiments (2), would allow a clear separation of the flow signal from the ECG. Neither technique was used in the present study.

The form of the external flowgram (Figures $3 B$ and 4) resembled the well-known left ventricle ejection curve with a steeply ascending and gradually descending branch. Not much can be learned from the rough outlines of this curve. However, the peaks and notches which appear in great regularity in the $X$ and $Z$ tracings (Figure $3 B$ ) offer most interesting information and a challenging new approach to a discussion of the electrical and mechanical events in cardiac activity. 
Cardiac Blood Flow During Isometric Contraction

The QRS complex was not quite completed at point A (Figure 4) when the flow potential started to rise. For all practical purposes, point $A$ corresponded on the timescale to point S of the ECG, as an extension of line R-A to the baseline demonstrates. The potential rose steeply in $10 \mathrm{msec}$ until point $B$ was reached. It is postulated that this part of the curve $(A-B)$ indicates movement of the blood in the heart during the isometric cardiac contraction and that point $B$ indicates the opening of the aortic valve.

The period A-B ( $10 \mathrm{msec}$ ) represents 4 per cent of the total time of one cardiac cycle of the squirrel monkey $(240 \mathrm{msec})$. In man the aortic valve opens about $30 \mathrm{msec}$ after point $S$ of the ECG, which is 3.75 per cent of the total cardiac period $(800 \mathrm{msec})$. Thus, it appears justified to associate point B of the flowgram with the opening of the aortic valve.

Other explanations of the discontinuity of the flowcurve at point B were considered and discarded: Branching of the blood stream at the root of the truncus brachiocephaliens, which is parallel to the magnetic field, would reduce the flow signal at point B. However, the branching point is anatomically so closely approximate to the peak of the aorta that the time period of $20 \mathrm{msec}$ appears too long for movement of the pressure wave between these two landmarks ( $B$ and $D)$. Furthermore, extension of the line from $B$ to beyond $A$ to the isoelectric line would place the starting point of the flow emf and thus the opening of the aortic valve well into the QRS complex. Opening of the aortic valve in the squirrel monkey at such an early time in the cardiac cycle appears most unlikely.

The electromotive force vector during the period of isometric heart contraction (A-B in Figure 4) was directed anteriorly and to the left of the animal, indicating that the blood in the heart during this period had a component of movement directed to the left and posteriorly. This is about the same direction as that found for the blood flow in the aorta. Under the assumption that a general cardiac contraction was in the direction apex to base during the isometric phase, most of the blood flow would be directed in the body axis and would not be registered in the magnetic field. A lateral component would have been expected to register to the right and not to the left as was actually seen.

The concept of an absolutely isometric contraction of the ventricle during the first part of ventricular systole was clarified by Rushmer's experimental work on systolic deflections of cardiac walls. Rushmer (6) found that "a considerable quantity of blood must be displaced within the enclosed ventricular cavity to produce these large early systolic deflections." It is this displacement of blood which is most probably recorded as a sharp upstroke of the A-B segment in Figure 4. While cardiac pressure and displacement measurements have been made numerous times during the period of "asynchronous contraction" (6-8), actual flow of blood in the heart during this period could be demonstrated by the present method for the first time. 
It should be noted in Figure 4 that the flow signal did not drop to zero before the aortic valve opened at point $B_{\text {; }}$ thus, the blood flow in the heart during asynchronous contraction continued into the aorta. This observation opposes the presently accepted theory of left ventricular ejection into the aorta which states that the blood starts its movement at the aortic valve from zero velocity and is accelerated rapidly by the pressure differential between ventricle and aorta. The flowgraphs of the present study suggest that a pulse wave was initiated in the ventricle during the "isometric" phase of contraction, reached the aortic valve, opened the valve, and passed into the aorta. Rushmer depicted ventricular ejection as a piston striking a mallet. According to that picture the piston is first struck, not at the opening of the aortic valve, but earlier in the left ventricle. Numerous flowgrams of other authors $(7,8)$, which show aortic flowgraphs starting from zero, are not in contradiction with the present view since the aortic flowmeters register the passage of the pulse wave at the site of the meter. Information on the origin of the pulse wave cannot be obtained with the conventional flowmeter method. By the magnetic method using external electrodes flow of blood in the heart as well as in the large vessels is registered, and a continuous record which could be easily timed by the superimposed ECG is provided.

Arterial and Venous Blood Flow

The slight decrease of the emf in section B-C was probably caused by the entry of the blood into the ascending branch of the aorta, which was practically parallel to the magnetic field and thus would not contribute to signal generation. Narrowing of the lumen of the blood column from the diameter of the heart (about $1.0 \mathrm{~cm}$ ) to the diameter of the aorta $(0.5 \mathrm{~cm})$ would also reduce the flow signal strength at point $B$.

The emf increased again as the blood reached the arch of the aorta and passed through a maximum at point $D$ while the blood flowed through the highest point of this vessel. At point $D$ the blood had a direction as indicated in Figure 8; this direction was perpendicular to the electrical flow signal illustrated in Figure 5. The flow points posteriorly with a small deviation to the left, as expected from the direction of the aortic arch.

From point D (Figure 4) the signal decreased as more and more blood passed through the descending branch. The singularity in this section at point $E$ could not be explained. The next peak on the downward slope of the flow curve was identified as the $T$ wave superimposed on the flowgram. The aortic valve closed, probably shortly before point $F$, and the steep descent to $F$ may be connected with the backflow of blood after closure of the valve. The peak of flow potential at point $G$ is probably connected with blood flow in the inferior vena cava where a maximum of flow occurs in early diastole.

The flow signal superimposed on the $P$ wave was most probably generated by venous return and filling of the left ventricle starting at point $\mathrm{H}$ (Figure 4) with opening of the mitral valve and initiation of the rapid filling phase. The strong dependence of this flow signal on the respiratory cycle was probably connected to the small pressure 
differential between left atrium and left ventricle characteristic for this phase. An increase of pressure in the chest cavity during expiration would be expected to decrease the velocity of filling of the left ventricle.

Thus the method gives information on arterial as well as venous blood flow in addition to the ECG. The following tongue twister designation for the records may be appropriate: Electro-magneto-cardio-arterio-venogram (EMCAVG).

New Quantitative Method to Determine the Velocity of Aortic Blood Flow

Under the assumption that point $B$ in Figure 4 indicates the opening of the aortic valve, an interesting direct method of determination of the flow velocity in the ascending branch of the aorta is suggested: According to the data in Table I the pulse wave travels from point $B$ to $D$ in $0.02 \mathrm{sec}$ and advances during this time from the aortic valve to the peak of the aortic arch. This distance was measured in aortograms of several monkeys and found to be $2 \mathrm{~cm}$. Thus, the pulse wave travels $2 \mathrm{~cm}$ in $0.02 \mathrm{sec}$, which corresponds to a mean pulse wave velocity of about $100 \mathrm{~cm}$ per sec in the ascending branch of the aorta of the squirrel monkey. The results of this method were based exclusively on a time and a distance measurement, the basic components of a velocity determination. The literature offered no values of the blood velocity in the aorta of small animals of less than $1 \mathrm{~kg}$ weight. Spencer and Greiss (7) found a range of maximum velocities from 52 to $150 \mathrm{~cm}$ per sec in dogs of a mean weight of $17 \mathrm{~kg}$. Hence, the velocity of $100 \mathrm{~cm}$ per sec found in the squirrel monkey by the magnetic method fell within a reasonable range.

Flow Dipole Used as Electrical Reference Source

An equation given by Kolin (9), that is,

$$
V=H \cdot d \cdot v \cdot 10^{-8}
$$

can be used to calculate the electrical potential generated on the walls of the aorta of a squirrel monkey. With a diameter $\mathrm{d}$ of the aorta of $0.5 \mathrm{~cm}$, a magnetic field $\mathrm{H}$ of $100,000 \mathrm{Oe}$ and a blood velocity $v$ of $100 \mathrm{~cm}$ per sec, the potential in volts was calculated as follows:

$$
V=10^{5}\left(5 \times 10^{-1}\right) \cdot 10^{-2} \cdot 10^{-8}=0.05
$$

With external electrodes a maximum flow potential of $0.75 \mathrm{mV}$ was found experimentally (Figure 7). Thus, the externally measured flow potential is considerably lower than the emf of $50 \mathrm{mV}$ calculated by the formula of Kolin for the wall of the aorta (factor 1 to 70).

With the site of the aorta and the site of the heart about equidistant from the surface of the body, the factor 1 to 70 found in the flow measurements was used to calculate the emf at the site of the heart from the amplitude of the $R$ wave in the frontal 
plane ECG $(1.6 \mathrm{mV})$. A value of about $110 \mathrm{mV}$ was found which is of the order of the action potential of the ventricular membranes. The principle of this approach may be of more general interest: The magnetic field in connection with pulsating blood flow allows generation of an artificial pulsating electrical dipole of known direction and strength inside the chest cavity, and other electrical activity in this cavity can be studied in reference to the flow dipole.

A comparison of the action potential and the flow potential at the site of generation, along with the corresponding values measured externally, is given in Figure 9. It is readily seen that the emf of the flow potential has a constant relationship with the externally measured signal. The flow potential is a current-supporting source during the total period of action. The action potential is current supporting only during its periods of main change which are measured externally as the $R$ and $T$ wave. This schematic comparison presents nothing principally new, but it may help to understand and illustrate the relationship of the action potential to the externally measured ECG.

\section{POSSIBLE CARDIAC STIMULATION BY THE FLOW POTENTIAL}

The flow potential which reached about $50 \mathrm{mV}$ at its source in the heart and the large vessels, and is a current-supporting source of sufficient duration, should be considered a potential cardiac stimulus. However, no sinus arrhythmia or extrasystoles were observed in short-duration experiments with monkeys. The fact that the main part of the flow potential fell into the refractory period of the heart obviously prevented a direct stimulation. The process of the repolarization of the monkey heart, as indicated by the $T$ wave, also was not noticeably influenced by the flow potential. However, the shape of the $T$ wave could not be determined with sufficient accuracy to exclude completely a possible effect of flow potential on repolarization.

In a previous study (1) where the squirrel monkeys were exposed to magnetic fields up to 70,000 Oe for a period of about one hour, a decrease of heart rate was observed. It appears possible that, during longer exposure to the magnetic field, the flow potential stimulated the aortic and the carotid sinus pressoreceptors which, via vagal branches and the parasympathetic system, eventually decreased the heart rate. The emf of blood flow was generated so closely to the pressoreceptors that an influence of this pulsating stimulus on the regulation of the heart rate seems possible. In future experiments an attempt will be made to study this more extensively.

The experiments with squirrel monkeys were performed in preparation for a systematic study of the possible effects of strong magnetic fields on man. Even though man probably will not be exposed in the laboratory or by industrial magnets to the extreme fields used in the present study, the results obtained from studying lower primates in very strong magnetic fields should help in the evaluation of possible hazards of strong magnetic fields to humans. With regard to future human exposure it is reassuring to know that the flow potential generated in the magnetic field by cardiac and large vessel blood flow did not immediately affect the cardiac activity of the experimental animals. However, in long-term human exposure to strong magnetic fields heart rate and blood pressure should be observed carefully. 


\section{REFERENCES}

1. Beischer, D. E., and Knepton, J. C., Jr., Influence of strong magnetic fields on the electrocardiogram of squirrel monkeys (Saimiri sciureus). Aerospace Med., 35:939-944, 1964.

2. Togawa, T., Okai, O., and Ohima, M., Observation of blood flow e.m.f. in externally applied strong magnetic field by surface electrodes. Med. biol. Engng., 5:169-170, 1967.

3. Laurence, J. C., Superconductive magnets at Lewis Research Center of NASA. NASA TM X-52269. Washington, D. C.: National Aeronautics and Space Administration, 1967.

4. Frank, E., An accurate, clinically practical system for spatial vectorcardiography. Circulation, 13:737-749, 1958.

5. Beischer, D. E., and Knepton, J. C., Jr., The electroencephalogram of the squirrel monkey (Saimiri sciureus) in a very high magnetic field. NAMI-972. NASA Order No. R-39. Pensacola, Fla.: Naval Aerospace Medical Institute, 1966.

6. Rushmer, R. F., Initial phase of ventricular systole: Asynchronous contraction. Amer. J. Physiol., 184:188-194, 1956.

7. Spencer, M.P., and Greiss, F. C., Dynamics of ventricular ejection. Circulation Res., 10:274-279, 1962.

8. Rushmer, R. F., Initial ventricular impulse: A potential key to cardiac evaluation. Circulation, 29:268-283, 1964.

9. Kolin A., Improved apparatus and technique for electromagnetic determination of blood flow. Rev. Sc. Instr., 23:235-242, 1952. 


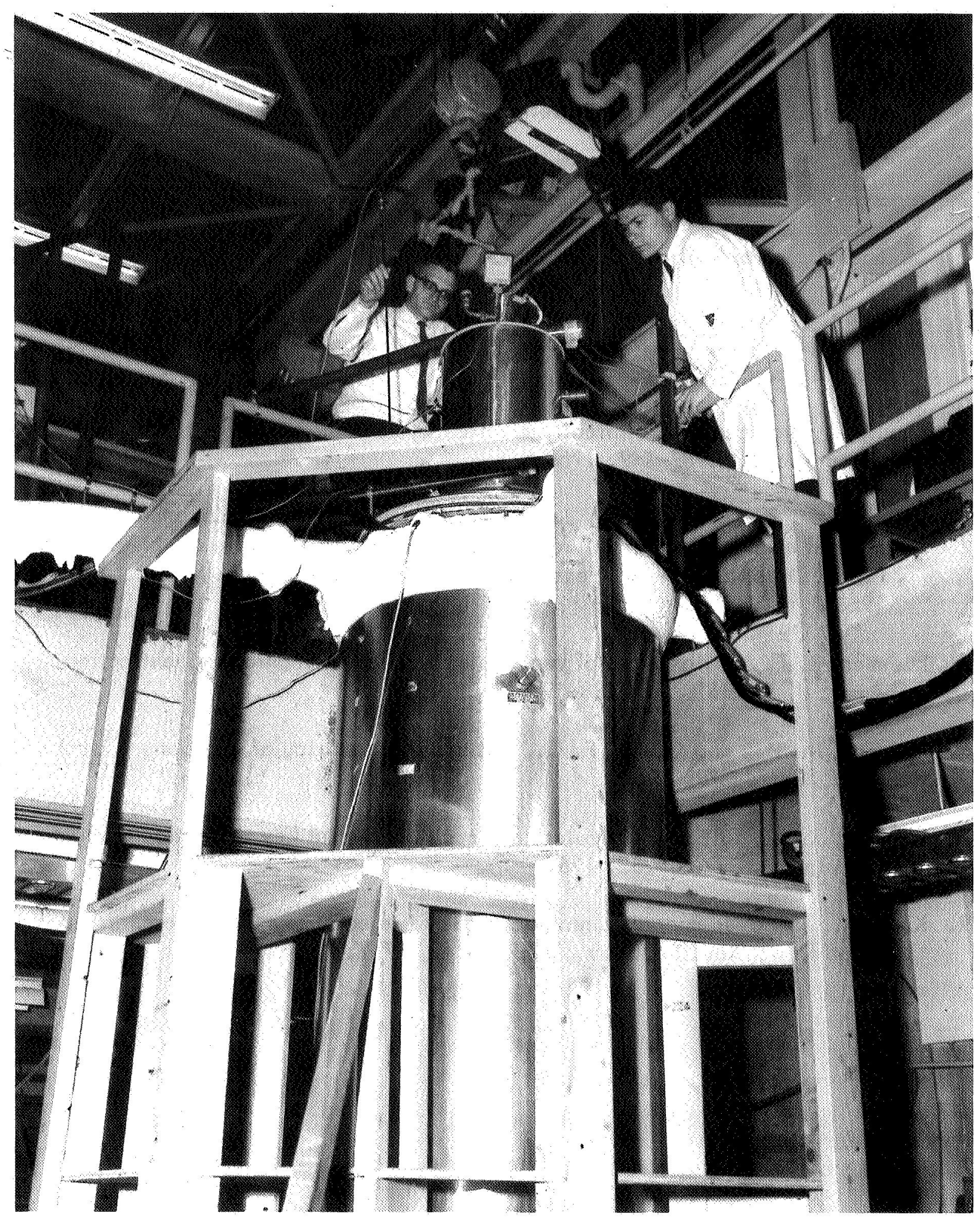

Figure 1

RCA superconductive electromagnet at Lewis Research Center, Cleveland, Ohio. Outside Dewar is snow covered on top. Operators look at opening of inside Dewar. 

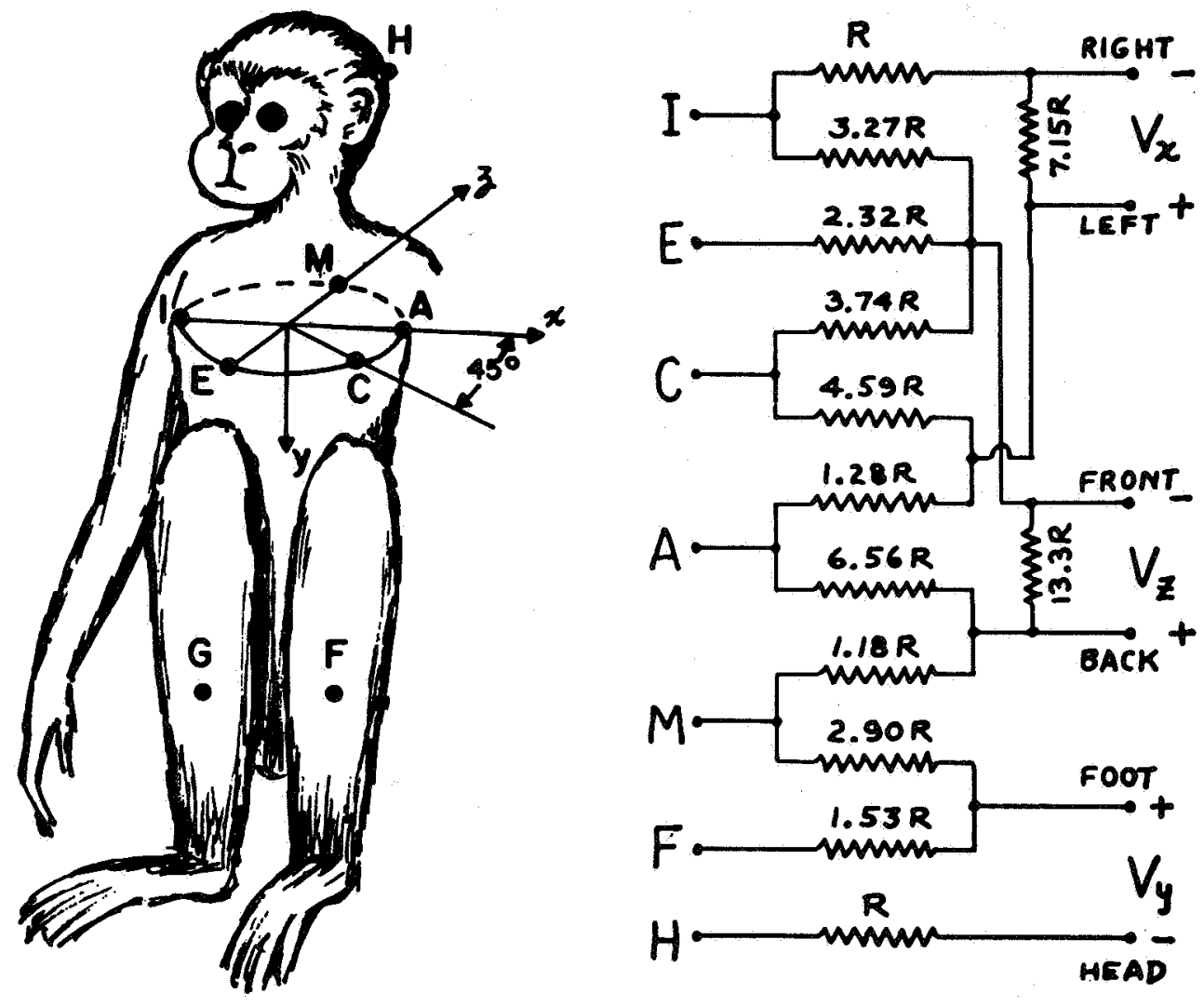

Figure 2

Frank lead system of vectorcardiography as applied to squirrel monkey. The same resistor proportions with reference to $R=100,000 \mathrm{ohm}$ as proposed by Frank for man were used for the monkey. Letters $A$ through $M$ designate Frank electrodes, and their placement is indicated on the depicted monkey. (Figure adapted from Frank (4)). 


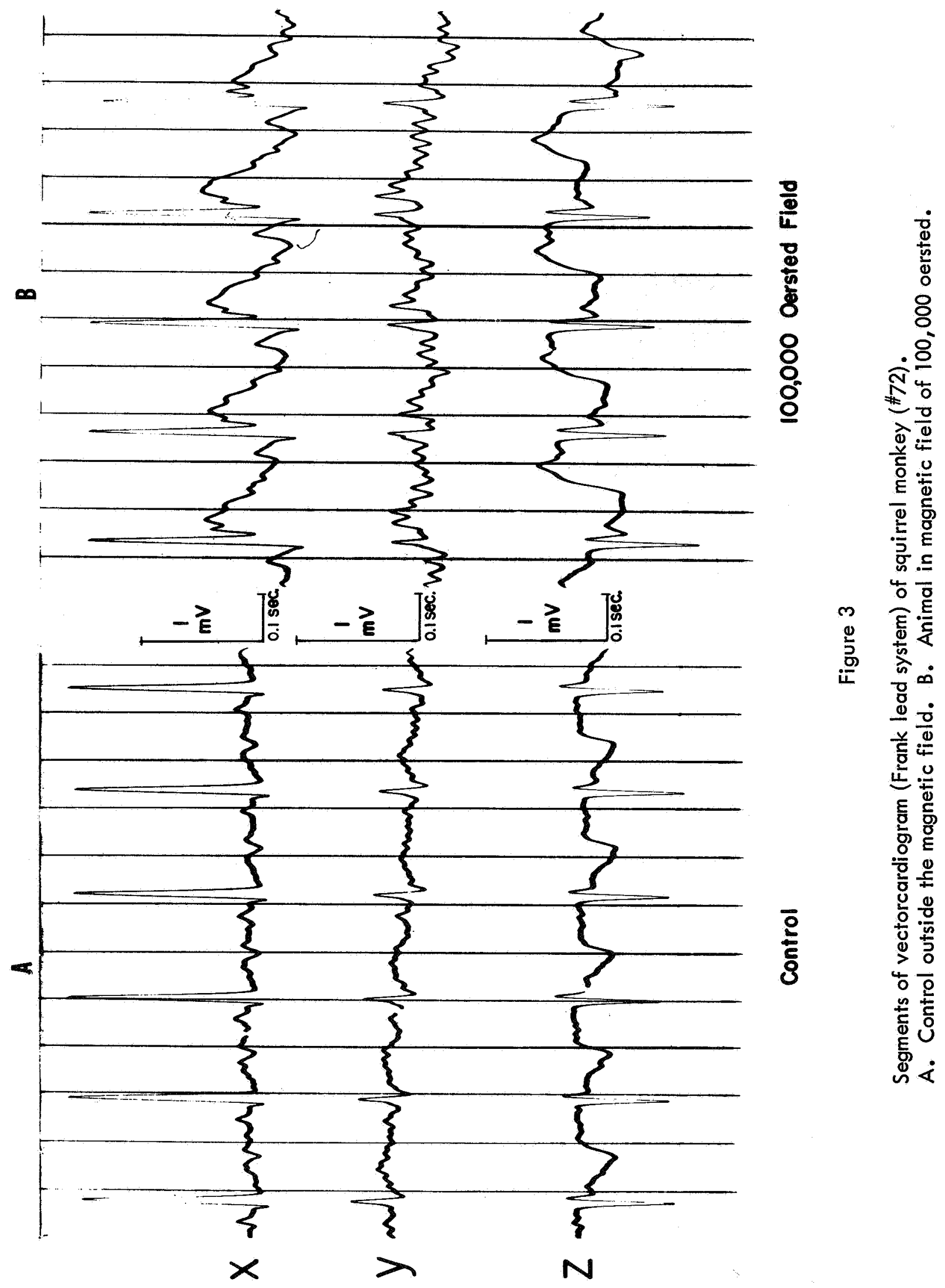




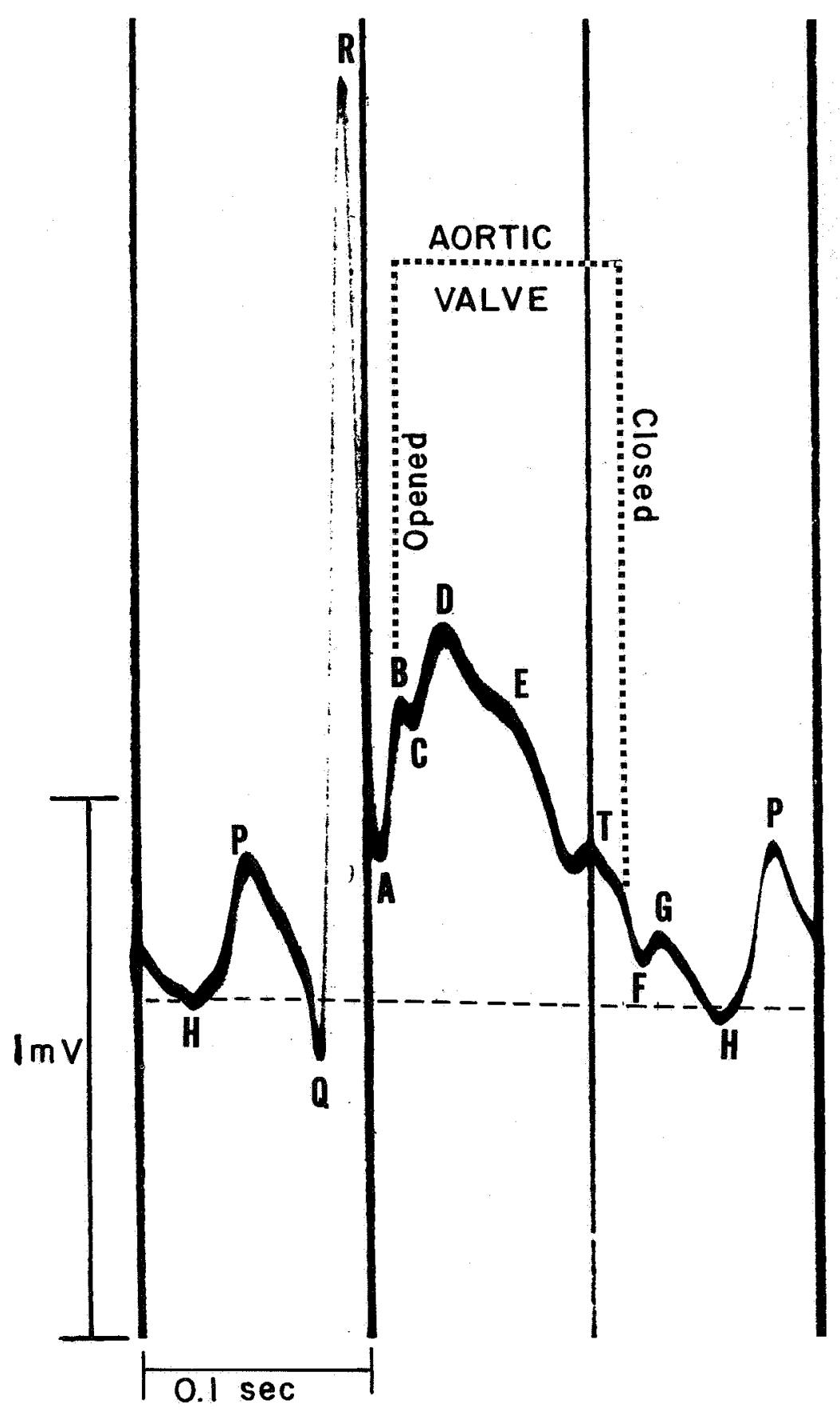

Figure 4

Single complex of $X$ tracing of frontal plane electrocardiogram (single points $P, Q, R, S$, and $T$ ) with superimposed flowgram. (See text pages 9 and 10 for explanation of points $A$ through $H_{\text {.) }}$ ) 


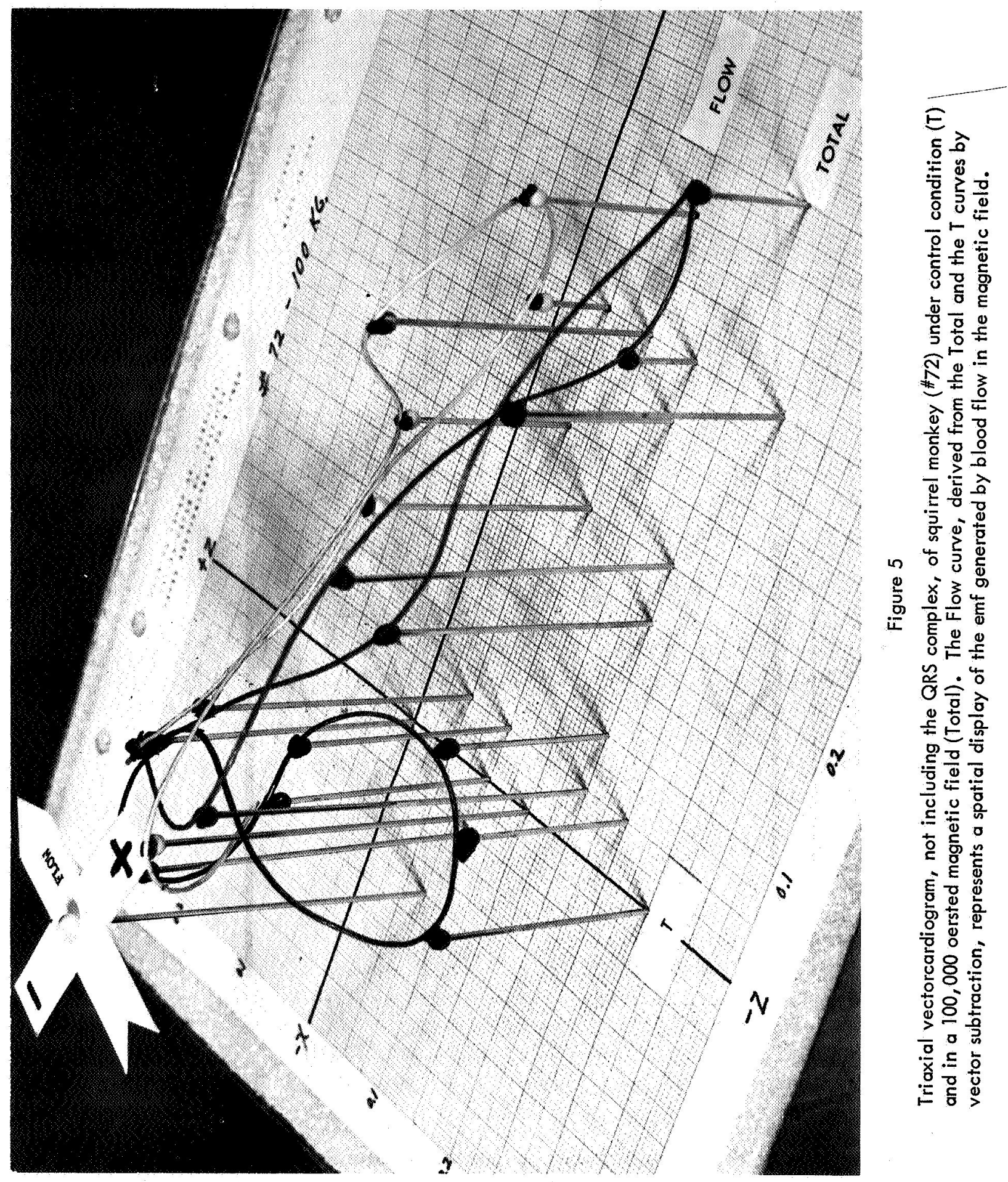




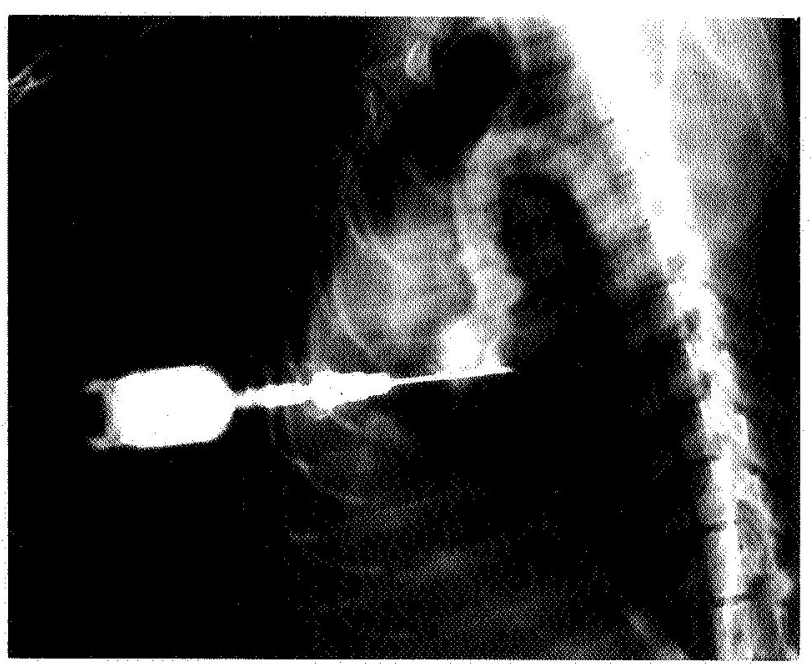

Figure 6

Normal aortogram (sagittal plane) of a squirrel monkey (prepared by Dr. H. H. Khalil). Distance from root of aorta to peak of aortic arch was $2 \mathrm{~cm}$.

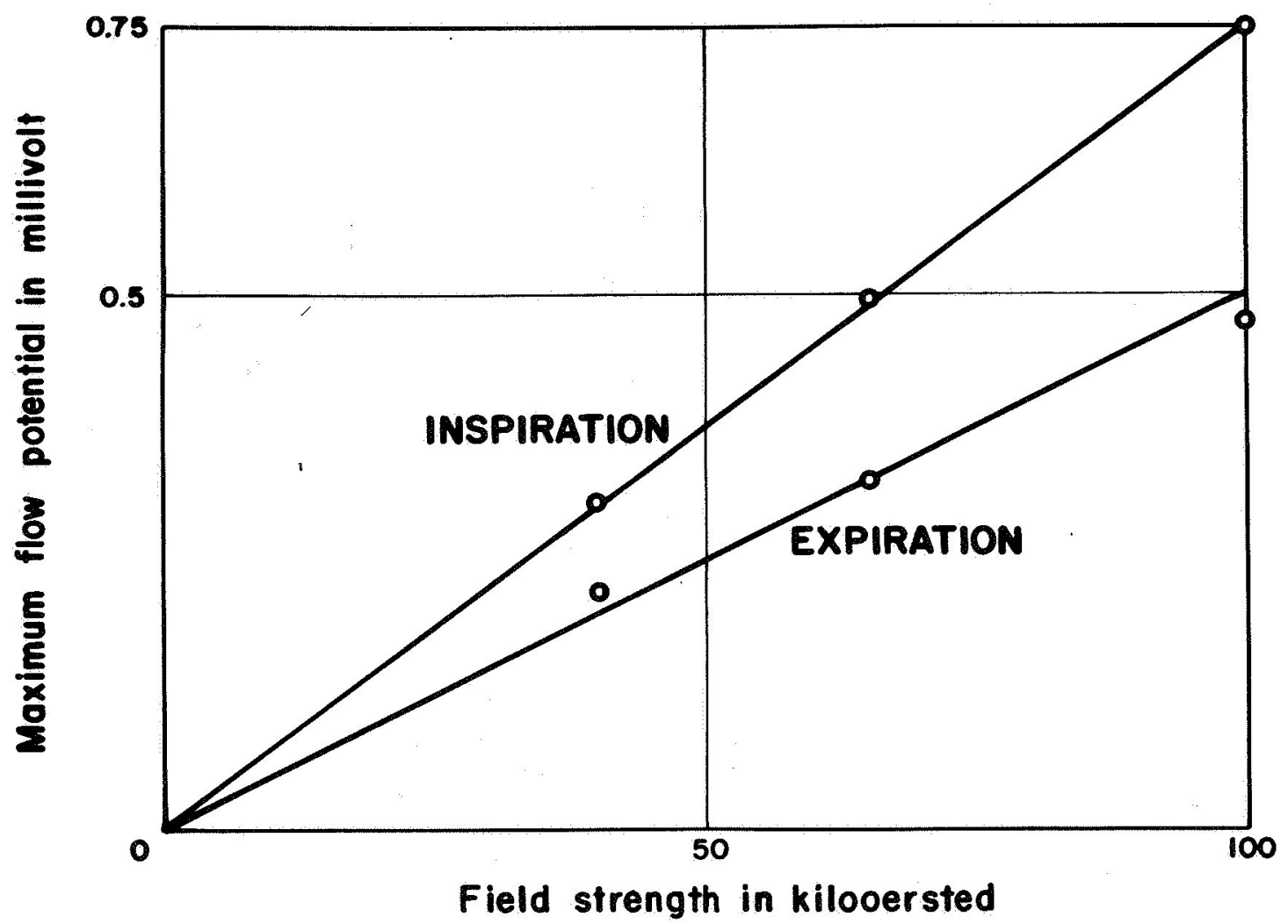

Figure 7

Maximum flow potentials generated by the different field strengths $(40,65,100 \mathrm{kOe})$ in the part of the aorta during inspiration and expiration. 


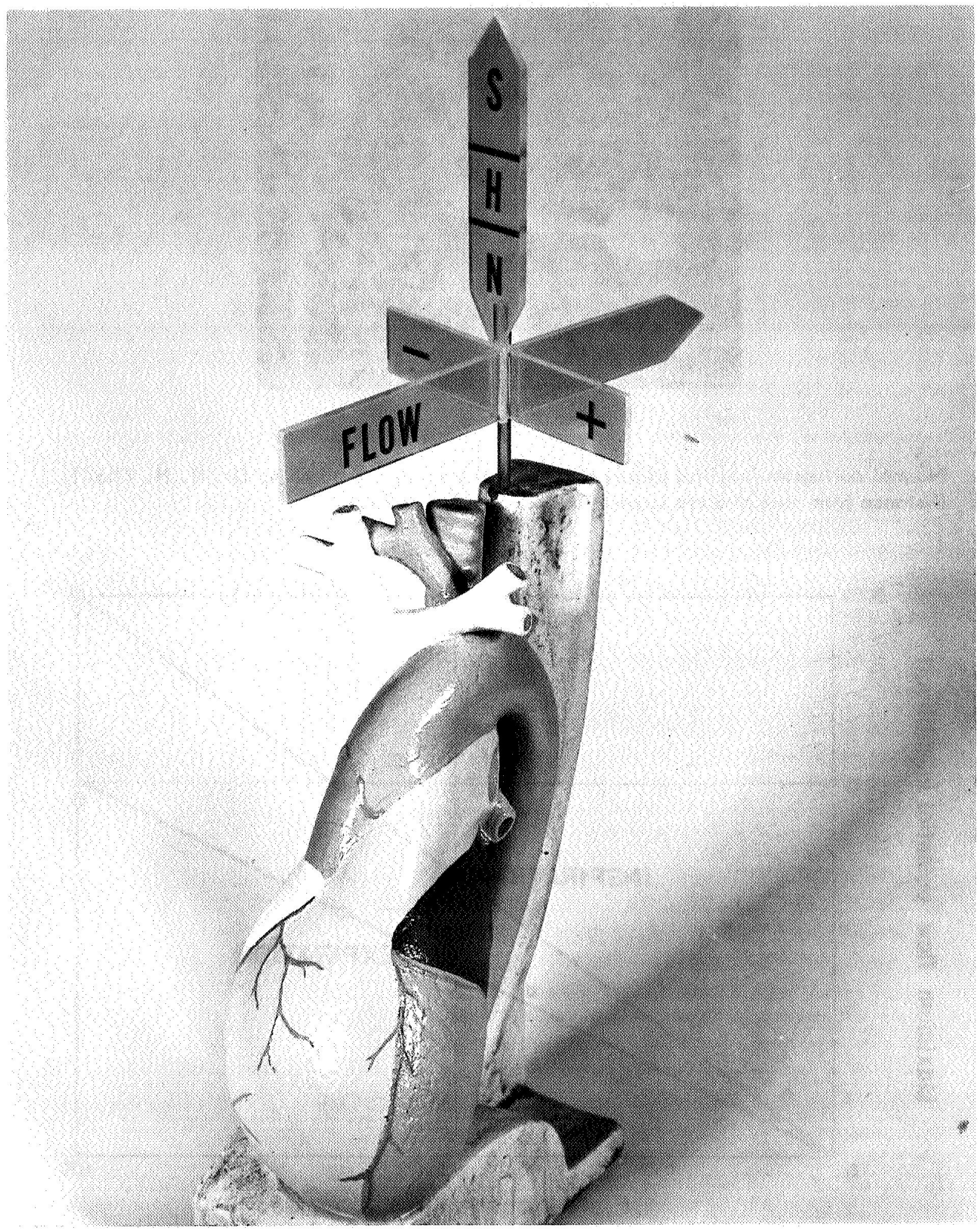

Figure 8

Electric dipole generated on the aortic wall by blood flow in a magnetic field. Using the right-hand rule the direction and polarity of the electrical dipole can be derived from the direction of blood flow in the aorta and the direction of the magnetic field $H(N-S)$. 

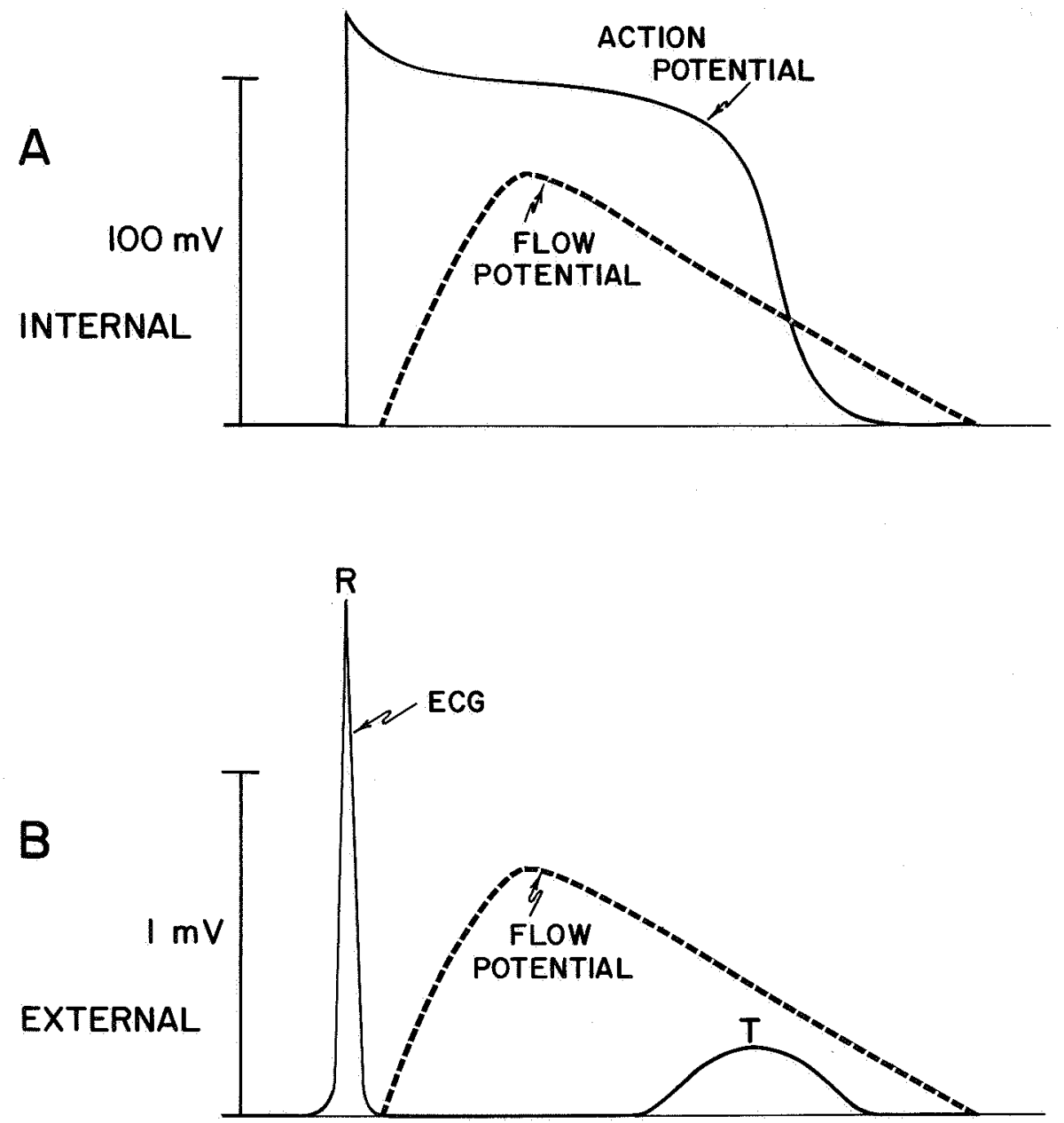

Figure 9

Relationship between internal (A) and surface values (B) of the cardiac potential ( - ) and the flow potential (--). 
Unclassified

Security Classification

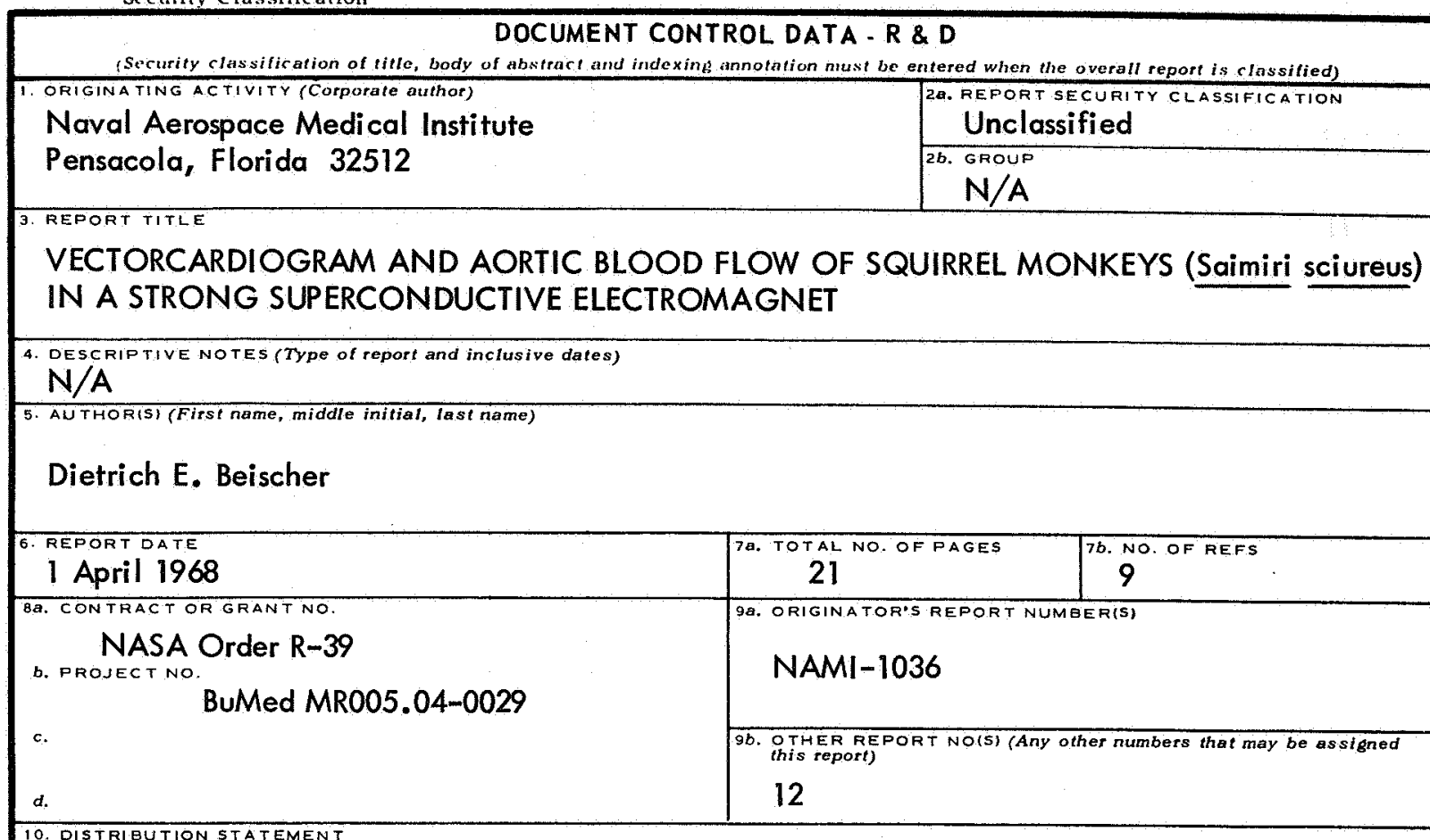

This document has been approved for public release and sale; its distribution is unlimited.

\begin{tabular}{|l|l|}
\hline 11. SUPPLEMENTARY NOTES & 12. SPONSORING MILITARY ACTIVITY \\
N/A & N/A \\
\hline 13. ABSTRACT & \\
\hline
\end{tabular}

In the future, strong magnetic fields will play a greater role in the environment of man. In preparation for this the frontal plane ECG of subhuman primates in strong magnetic fields (100,000 oersted) was recorded. A pulsating electrical signal (flowgram) was found superimposed on the ECG, giving information on mechanical as well as electrical performance of the heart. No influence of the magnetic field on cardiac regulation was observed during short exposure periods. 
Unclassified

Security Classification

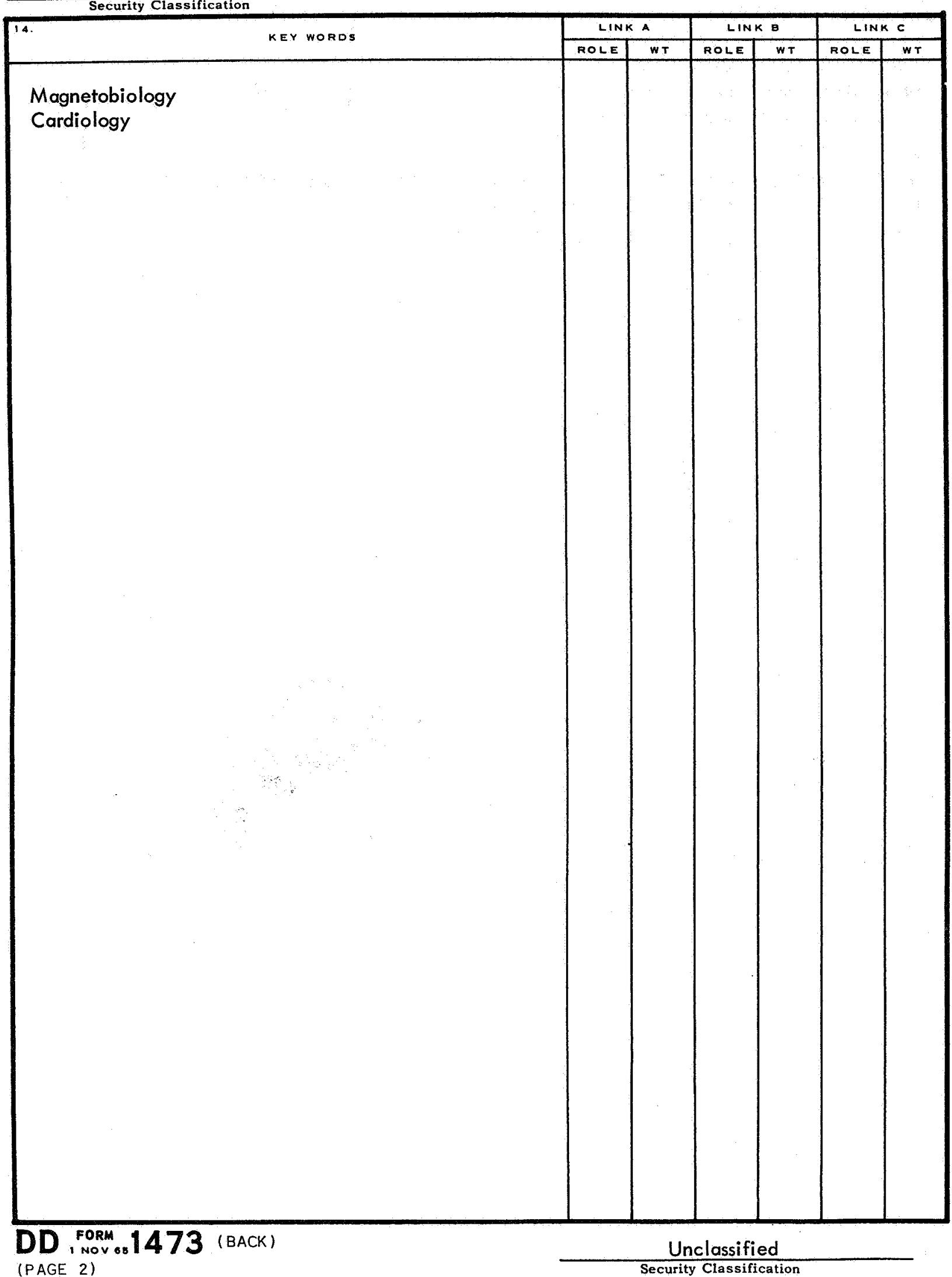

